\title{
Covid-19 - um exercício de coordenação e articulação municipal efetiva: a experiência de Niterói
}

\author{
Covid-19 - an effective municipal coordination and articulation \\ exercise: the Niterói experience
}

Mirian Ribeiro Conceição', Renata Pascoal Freireㄹ, Filipe Côrtes Macario², Rodrigo Alves Torres

Oliveira², Camilla Maia Franco 2

DOI: 10.1590/0103-11042020E419

RESUMO Este artigo visou apresentar a experiência do município de Niterói na criação e na coordenação das ações de enfrentamento da Covid-19. O planejamento e a implementação das diferentes estratégias foram embasados na intersetorialidade como linha transversal de gestão, considerando, a partir dos fatores de impacto da pandemia na população, não apenas o aspecto unidimensional de responsabilidade do âmbito clínico da Covid-19, mas também a complexidade do cuidado das condições de vulnerabilidade, dos impactos socioeconômicos das medidas de mitigação e dos determinantes sociais da saúde. Para tal, a construção de um modelo de gestão intersetorial, com a criação de um Gabinete de Crise formado pelos representantes de diferentes secretarias, resultou em ações que objetivaram responder às necessidades pertinentes à complexidade da vida, na comunicação contínua com a população garantir o acesso a informações e a adesão da sociedade, fator central no êxito das medidas de mitigação.

PALAVRAS-CHAVE Infecções por coronavírus. Colaboração intersetorial. Saúde pública. Sistema Único de Saúde. Gestão em saúde.

ABSTRACT This article aims to present an experience of the city of Niterói regarding the creation and coordination of coping actions for Covid-19. The planning and implementation of the different strategies was based on intersectoriality as the transversal management line, considering, based on the impact factors of the pandemic on the population, not only the one-dimensional aspect of responsibility of the clinical studies of Covid-19, but also the treatment vulnerability conditions, socioeconomic impacts of mitigation measures, and social determinants of health. For such, the construction of an intersectoral management model with the creation of a Crisis Office formed by representatives of different secretaries resulted in actions that aimed to respond to the specific complex needs of life, with continuous communication with the population in order to guarantee access to information and the support of society, a central factor in monitoring mitigation measures.

KEYWORDS Coronavirus infections. Intersectoral collaboration. Public health. Unified Health System. Health management.

1 Universidade Federal

Fluminense (UFF) - Niterói

(RJ), Brasil.

mirianrcon@gmail.com

2 Secretaria Municipal de

Saúde (SMS) - Niterói

(RJ), Brasil. 


\section{Introdução}

$\mathrm{O}$ aumento de casos de pneumonia, no final de dezembro de 2019, na cidade de Wuhan República Popular da China - criou um alerta às autoridades locais e à Organização Mundial da Saúde (OMS). O vírus identificado como SARS-CoV-2, o novo coronavírus, responsável pela doença Covid-191, teve rápida propagação da infecção com nível de alerta alto de atenção das autoridades mundiais. A difusão da Covid19 para diferentes países, com mais de 118 mil casos confirmados e 4.291 mortes até 11 de março de 2020, definiu o caráter pandêmico da doença ${ }^{2}$. Tais alertas mobilizaram os governos de todo o mundo para se organizarem para enfrentamento da situação.

Em âmbito nacional, o Ministério da Saúde (MS) definiu o monitoramento da doença no Brasil a partir da adoção de uma ferramenta de classificação com três níveis - Alerta, Perigo Iminente e Emergência em Saúde Pública - que determinaria as ações a serem desenvolvidas, seguindo a mesma linha utilizada globalmente na preparação e resposta em todo o mundo ${ }^{3}$.

O primeiro caso registrado no Brasil foi em 26 de fevereiro de 2020, iniciando-se em março os primeiros casos notificados de transmissão comunitária. Estados e municípios começaram seus planos de enfrentamento da pandemia pautados principalmente em medidas de contenção e mitigação dos contágios. Estimar a progressão do processo epidêmico era um grande desafio nesse início, haja vista as características do Brasil: o primeiro grande país com altas densidades populacionais em algumas regiões metropolitanas, de índice de desigualdade social elevado, com limites operacionais seja na capacidade de atendimento do Sistema Único de Saúde (SUS), seja do componente suplementar do Sistema.

Em alerta máximo de uma situação pandêmica, principalmente no que tange à prevenção e ao tratamento de doenças infecciosas como a Covid-19, criou-se uma percepção unidimensional de responsabilidade do âmbito clínico da doença ${ }^{4}$. Entretanto, as condições de vulnerabilidade, o impacto socioeconômico das medidas de mitigação e os determinantes sociais da saúde convocaram governantes e gestores à construção de planos de enfrentamento pautados na complexidade da vida e nos diferentes determinantes da saúde.

Nesse sentido, considerar a construção de políticas específicas e a proteção de grupos mais vulneráveis foi decisivo no enfrentamento da pandemia. Estudos como o do Imperial College - The Global Impact of Covid-19 and Strategies for Mitigation and Suppression ganharam destaque nas progressões e análise do impacto nos cenários globais considerando as estratégias de mitigação. Para os autores, a ausência de medidas de mitigação - como proteção do grupo de risco, como a população idosa -, bem como de estratégias que retardam a transmissão poderiam reduzir pela metade o número de óbitos, sinalizando maior severidade do impacto da pandemia em cenários de baixa renda, com maior tendência de colapso dos sistemas sanitários, e, portanto, reforçando a precocidade e a antecipação das estratégias de mitigação ${ }^{5}$.

O mesmo estudo apresentou algumas projeções representadas por três cenários por região do Banco Mundial: 1. Uma epidemia não mitigada - um cenário no qual nenhuma ação é tomada; 2. Implementação de medidas de mitigação incluindo o distanciamento social; e, 3. Mitigação incluindo maior distanciamento social dos idosos ou reduzindo suas taxas de contato social em $60 \%$.

Desse modo, a precocidade da implementação das ações de contenção e mitigação, sobretudo em países com maior vulnerabilidade social, seria o cenário mais favorável para evitar mortes e o impacto social da Covid-19.

Este artigo, portanto, visa apresentar a experiência do município de Niterói na criação e coordenação das ações de enfrentamento da Covid-19, tendo a intersetorialidade como linha transversal de planejamento e gestão, a partir dos fatores de impacto da pandemia na população, principalmente na faixa mais vulnerável. 
Considera-se, para esta análise, a pluralidade do vocábulo 'intersetorialidade', no âmbito da saúde ${ }^{6}$, conceituada por alguns autores como 'a práxis da articulação de saberes e experiências, que com objetivos comuns buscam para solução sinérgica de problemas complexos $^{\text {7-11. }}$. Desse modo, as diferentes ações implementadas no município foram coordenadas por saberes diversos para criar respostas integrais e resolutivas para a complexidade do enfrentamento da pandemia, não apenas no âmbito clínico como também no atendimento às demandas para o sistema sanitário, na construção de respostas aos efeitos das medidas de mitigação.

A reconstrução das ações e a análise dos resultados apresentados neste relato de experiência foram coletadas a partir da revisão de documentos - decretos e portarias -; bem como da vivência dos autores no âmbito da gestão municipal, no período de janeiro a junho de 2020.

\section{Planejamento e gestão do plano de enfrentamento da covid-19 no município de Niterói}

\footnotetext{
O município de Niterói, campo de referência para este estudo, iniciou as estratégias de enfrentamento de modo precoce, coordenando ações de isolamento social, bem como de mitigação dos seus efeitos sociais e econômicos, com ampliação e desenvolvimento da capacidade de atendimento em saúde e grande investimento na comunicação com os munícipes.

A cidade de Niterói faz parte da Região Metropolitana II do estado do Rio de Janeiro, composta pelos municípios de Itaboraí, Maricá, Rio Bonito, São Gonçalo, Silva Jardim e Tanguá. Na estimativa do Instituto Brasileiro de Geografia e Estatística (IBGE) para 2019, o município tem população de 513.584 habitantes, sendo o quinto em população do estado e o segundo da Região Metropolitana II, representando $24,57 \%$ da região ${ }^{12}$.
}

Em destaque para o cenário de enfrentamento da pandemia, $100 \%$ da população reside em área urbana e tem o crescimento significativo da população idosa em relação à população geral nos últimos anos. Segundo dados do Departamento de Informática do SUS (Datasus/MS), a população de Niterói acima de 60 anos, em relação à população geral, aumentou de $13,1 \%$ no ano de 2000 para 19,5\% em 2015 em relação à população geral. Um incremento de $48,8 \%$ na população acima de 60 anos em apenas 15 anos $^{13}$.

O município registrou seu primeiro caso de Covid-19 no início de março, mas antecipou parte de suas ações, pautadas nas experiências de outros países, ainda em janeiro, quando foi anunciada pela OMS a situação de 'Emergência de Saúde Pública de Importância Internacional'14.

Uma das primeiras medidas realizadas no âmbito do planejamento e coordenação das ações pela Prefeitura de Niterói foi a criação do 'Grupo de Resposta Rápida' com o objetivo de fortalecer a capacidade do sistema de saúde do município para responder ao novo coronavírus. Desse modo, dedicou-se ao planejamento dos fluxos de encaminhamento, à reserva de leitos, às compras de Equipamento de Proteção Individual (EPI), às medidas de prevenção, capacitação e formação dos profissionais da rede, entre outras pautas fundamentais nas ações de contenção e enfrentamento da pandemia.

Na sequência das ações de gestão em modo intersecretarial, foram criadas, em 14 de março, duas estruturas: 0 Gabinete de Crise da Prefeitura de Niterói e a Sala de Situação da Secretaria Municipal de Saúde/Fundação Municipal de Saúde de Niterói com vistas à intensificação das estratégias de enfrentamento ${ }^{15}$. O Gabinete de Crise conta com a coordenação direta do Prefeito, tendo em sua composição as secretarias de governo - Secretarias de Saúde, Educação, Planejamento, Comunicação, Idoso, Ordem Pública, Administração, Fazenda, ProcuradoriaGeral, Urbanismo, Transportes e Mobilidade, Defesa Civil e Assistência Social; Secretaria Executiva; Secretaria Municipal de Saúde; Secretaria Municipal de Educaçãon.

$\mathrm{O}$ envolvimento das diferentes secretarias 
teve como principal objetivo o planejamento e a execução das ações de enfrentamento, considerando a complexidade do impacto sanitário, social e econômico e a diversidade das ações/ medidas de mitigação.

As ações planejadas e implementadas pela gestão pública se articulam com a prática de intersetorialidade, adotada por Sposati16 ao admitir uma decisão racional no processo de gestão, atuando de forma complementar às práticas setoriais, ou seja, realizando uma combinação entre política setorial com intersetorial, não contrapondo os processos de gestão.

Seguindo as recomendações internacionais e nacionais, sobretudo experiências técnicas reconhecidas cientificamente, foram instituídas medidas 'não farmacológicas': o isolamento social, a restrição à circulação das pessoas na cidade, o distanciamento de interpessoal, o afastamento de pessoas com sintoma da doença, a higiene regular das mãos e de objetos de uso frequente (assim como as práticas complementares de higiene) e etiqueta respiratória) ${ }^{\mathbf{1 7}}$.

As ações de saúde adotadas integravam-se a um conjunto de estratégias assumidas por outras áreas de governo. Baseada em experiências internacionais (Portugal, Itália Espanha) ${ }^{\mathbf{1 8 - 2 0}}$, as ações estruturadas pelo Gabinete de Crise foram de caráter intersetorial, pautadas por quatro eixos: isolamento social, mitigação dos efeitos sociais e econômicos da pandemia, saúde e comunicação, tendo a gestão como eixo transversal e condutor da tomada de decisão.

Nesse sentido, as ações de enfrentamento da Covid-19 foram pensadas para além das dimensões biológicas da doença, considerando que atuar sobre as condições sociais e na mitigação das desigualdades sociais teria uma maior determinação no processo saúde-doença e, com isso, maior êxito da estratégia²1.

As primeiras estratégias de isolamento social no município foram pautadas pela suspensão dos eventos culturais e esportivos e das atividades escolares na rede pública. A partir de 23 de março, a Prefeitura de Niterói decretou um conjunto de medidas restritivas no intuito de reduzir a circulação de pessoas, e com realização de isolamento total (lockdown) de 11 a 21 de maio, período que apresentava criticidade nas projeções epidemiológicas ${ }^{\mathbf{1 4}}$.

O conjunto de medidas restritivas adotadas incluiu a criação de barreiras sanitárias e restrição dos acessos a Niterói, além da suspensão das atividades de diferentes equipamentos públicos (museus, centros culturais, bicicletário e outros), suspensão das aulas na rede municipal de educação e de concursos públicos, interdição das praias e atividades comerciais não essenciais, fechamento do acesso às praias e criação de canais de denúncias para atividades contrárias às recomendações de isolamento.

As diferenças de renda influenciam nas condições de saúde devido à escassez de recursos dos indivíduos e à falta de infraestrutura comunitária $^{22}$. Nesse sentido, atuou-se de modo a responder às complexidades sociais com a implementação de ações intersetoriais nos âmbitos sanitários, sociais e econômicos. Estas foram operadas no âmbito da gestão em que as diferentes secretarias puderam, a partir das decisões necessárias ao enfrentamento no âmbito sanitário, pactuar com os interlocutores dos diferentes setores do campo atuação de suas políticas9. Desse modo, por meio de estratégias planejadas conjuntamente a partir do mapeamento das necessidades dos setores, foram desenvolvidos:

- Plano de Mitigação dos Impactos Econômicos e Sociais - Fundo Niterói Supera (parceria com banco público, para suporte de crédito para micro e pequenas empresas, profissionais autônomos e liberais, cooperativas e associações de produção) ${ }^{\mathbf{2 3}}$; Programa Empresa Cidadã (ajuda financeira da Prefeitura por meio do pagamento de um salário mínimo para até nove empregados de empresas, entidades religiosas e sindicais, de qualquer porte)24; Auxílio para Microempreendedores Individuais, Taxistas e Motoristas Autônomos (auxílio financeiro aos Microempreendedores Individuais) ${ }^{25}$.

- Criação da Câmara Temática da área econômica - pactuada pela Prefeitura de Niterói 
com entidades empresariais e industriais da cidade, com a função de acompanhamento planejamento coparticipativo para discutir medidas que pudessem reduzir o impacto na crise econômica no município.

- Grupo de Trabalho para retomada das atividades econômicas - com a atribuição de debater e elaborar conjuntamente com a prefeitura plano de ação para retomada das atividades, tendo em consideração a preservação da saúde dos munícipes. Grupo com periodicidade semanal composto por representantes municipais do setor econômico, e as secretarias municipais de: Administração, Desenvolvimento Econômico, Fazenda, Governo, Planejamento, Orçamento e Modernização da Gestão, Saúde, além de outras entidades, tais como: Associação Comercial, Associação Conselho Empresarial e Cidadania, Associação de Dirigentes de Empresas do Mercado Imobiliário, Câmara de Dirigentes Lojistas, Federação das Indústrias do Rio de Janeiro, Fundação Oswaldo Cruz, Sindicato dos Lojistas do Comércio, Universidade Federal do Rio de Janeiro e Universidade Federal Fluminense ${ }^{26}$.

No âmbito da Saúde, as ações da Secretaria Municipal de Saúde foram sistematizadas no Plano Municipal de Resposta à Pandemia do Coronavírus que incluiu um conjunto de recomendações no campo da prevenção e vigilância em saúde e reorganização da estrutura assistencial para intensificar os cuidados contra doenças de transmissão respiratória. $\mathrm{O}$ conjunto de medidas vem sendo atualizado de forma permanentemente a partir de novas evidências científicas, normativas ministeriais e, sobretudo, das análises epidemiológicas dos casos do município.

Dentre as estratégias adotadas no campo sanitário, para ampliação da capacidade de atendimento, cabe destacar o arrendamento de uma estrutura hospitalar, para instalação com recurso próprio para atendimento exclusivo de usuários em alto risco de SARS-CoV-2, contando com 140 leitos, com capacidade para diagnóstico de risco com radiologia digital e tomografia computadorizada, bem como aparelhos para ventilação mecânica e monitorização completa com oximetria e capnometria. Para além dessa unidade hospitalar exclusiva, foi ampliada a capacidade de atendimento das unidades de saúde existentes - Hospital Municipal Carlos Tortelli, Hospital Getúlio Vargas Filho e Hospital Universitário Antônio Pedro - para atendimento clínico e crítico aos casos adultos e infantis, totalizando em 262 leitos no município, distribuídos em 97 leitos clínicos e 165 críticos.

Cabe ressaltar que todos os leitos descritos foram ofertados seguindo fluxos e critérios técnicos definidos pela Central de Regulação Municipal, também reestruturada para funcionamento 24 horas e adequada às normativas vigentes, passando a ser uma estratégia vital para reordenamento e ampliação de acesso na cidade $^{27}$.

Outro ponto fundamental na capacidade de atendimento dos usuários pela rede de Niterói deu-se pela orientação e criação de fluxos específicos entre a rede de serviços da Atenção básica e as unidades de atendimento de emergência e unidades hospitalares, garantindo detecção, avaliação e tempo de resposta adequados ao cuidado e evolução da Covid-1928.

Desde o início da transmissão comunitária no município, houve especial atenção à capacidade de testagem para detecção dos casos em modo a conter a transmissão. Niterói foi o primeiro município brasileiro, a fazer testagem em massa de Covid-19, seguindo as recomendações da OMS, estratégia fundamental de contenção da pandemia ${ }^{29}$.

Com o objetivo de identificar as pessoas infectadas e garantir o isolamento social e a diminuição do comunicantes e de doentes assintomáticos, minimizando a propagação do novo coronavírus foram realizados ainda na primeira fase $40 \mathrm{mil}$ testes. Atualmente o município apresenta padrão de testagem semelhante aos países europeus, numa proporção de 40/1000 habitantes.

Foram atribuídas às unidades básicas e policlínicas, a ordenação dos fluxos de testagem de pacientes sintomáticos, bem como a realização 
das avaliações iniciais com estratificação dos casos, realização do manejo clínico para os casos leves, além da coordenação do cuidado para os casos que necessitavam de atenção mais especializadas ${ }^{30}$.

Por fim, foi organizado um processo seletivo em caráter emergencial que resultou na ampliação imediata da cobertura assistencial à população por meio da contratação de 456 profissionais de saúde para atuação na Rede Atenção à Saúde de Niterói ${ }^{31}$, com vistas à ampliação das estruturas, leitos e capacidade de atendimento para atuação na linha de cuidado e enfrentamento à pandemia.

Associada às medidas restritivas foram adotadas outras ações de prevenção, a fim de contribuir na diminuição do contágio, tais como: o uso obrigatório e a distribuição de máscaras e kits de higiene e sanitização de ruas e comunidades.

Outra ação intersetorial que impactou prioritariamente no cuidado da população mais vulnerável foi a construção de um Centro de Referência em Quarentena Assistida (CRQA). Criado em parceria entre a Secretaria de Saúde e a Secretaria de Assistência Social, o CRQA possibilitou isolamento social para usuários positivo com Covid-19 e estratificados como casos leves pela atenção básica ${ }^{28}$.

O centro foi destinado aos usuários que não dispunham de condições habitacionais. Com capacidade para acolher 120 pessoas, contou com uma equipe multiprofissional - educadores, assistentes sociais, psicólogos, enfermeiros e técnicos de enfermagem, médicos de suporte, que atuavam na promoção de ações de bem-estar e de monitoramento de sinais e sintomas, com vistas a garantir o cuidado adequado aos usuários. Vale ressaltar que a unidade era caracterizada como equipamento social, e não estava prevista nenhuma complexidade de cuidado em saúde para a estrutura; portanto, em caso de agravamento dos sintomas, a rede de cuidados em saúde oferecia tal suporte, permitindo, desse modo, total segurança para o usuário.

O CRQA foi dispositivo importante de suporte seja para o âmbito da saúde, seja para a assistência social. Esta última, na criação de estratégias de proteção e cuidado à população em situação de rua, pode, por meio do arrendamento de hotéis, abrigar usuários na região central da cidade com o objetivo de garantir a saúde, o isolamento social, a segurança e a alimentação das pessoas em tais condições. O dispositivo acolheu homens e mulheres, em parceria com as equipes de consultório na rua, que não estavam infectados pelo SARS-CoV-2. Em eventuais suspeitas de infecção, foi realizado fluxo prioritário com as unidades básicas de saúde para avaliação, testagem e encaminhamento para o CRQA.

\section{Algumas reflexões}

As estratégias elencadas anteriormente, nos âmbitos sociais, econômicos e sanitários, objetivaram o enfrentamento da pandemia, no campo do planejamento e gestão, tendo no Gabinete de Crise o espaço para definição de estratégias intra e intersetoriais. O diálogo contínuo das secretarias de governo com os diferentes setores, seja no compartilhamento das informações, seja no planejamento compartilhado de planos de ações que pudessem suplantar o impacto da pandemia, operou em modo prático a intersetorialidade, fortalecendo políticas e ações governamentais?.

Por fim, ponto fundamental no fortalecimento e na garantia da efetividade das ações planejadas e implementadas foram os dispositivos de comunicação que consistiram em um conjunto de ações com vistas a sensibilizar a população quanto à importância do seu envolvimento e contribuição no enfrentamento da pandemia. Para isso, foram usados diferentes mecanismos: mensagens diárias às comunidades sobre as ações da prefeitura no combate à pandemia; distribuição de cartilhas com informações para auxiliar na prevenção e reconhecimento de sintomas para detecção precoce da Covid-19; utilização de vídeos diários com notícias atualizadas; e a construção de sítios eletrônicos específicos para Covid-19.

Pode-se dizer que o conjunto de ações implementadas em companhia do engajamento da população nas ações de mitigação possibilitaram 
o êxito da principal estratégia de enfrentamento da Covid-19 utilizada pelos países em todo o mundo: o isolamento social. Observou-se a redução da circulação da população em índices superiores a $60 \%$, no período de maior incidência epidêmica (meses de março a maio), mantendo índices significativos no momento de retomada das atividades.

Gráfico 1. Histórico de isolamento social diário, Niterói, 2020

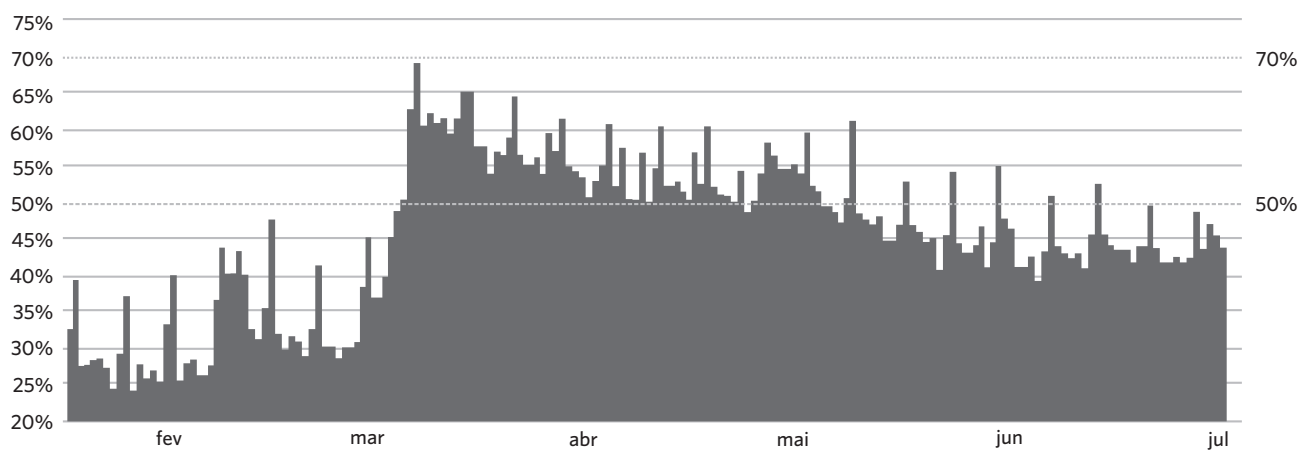

Fonte: Secretaria Municipal de Saúde de Niterói - SIGeo - Niterói33.

A análise dos dados da cidade de Niterói demonstra que ela atingiu rapidamente um patamar de isolamento em torno de $60 \%-65 \%$ logo no início da aplicação das medidas restritivas. Ademais, que as taxas permaneceram entre
$50 \%$ e $60 \%$ ao longo dos meses de abril e maio.

As medidas implementadas também trouxeram impactos significativos no cenário epidemiológico do município com importante decréscimo nos índices de novos casos e óbitos.

Gráfico 2. Distribuição de novos casos por semana epidemiológica por data de sintomas, Niterói, 2020

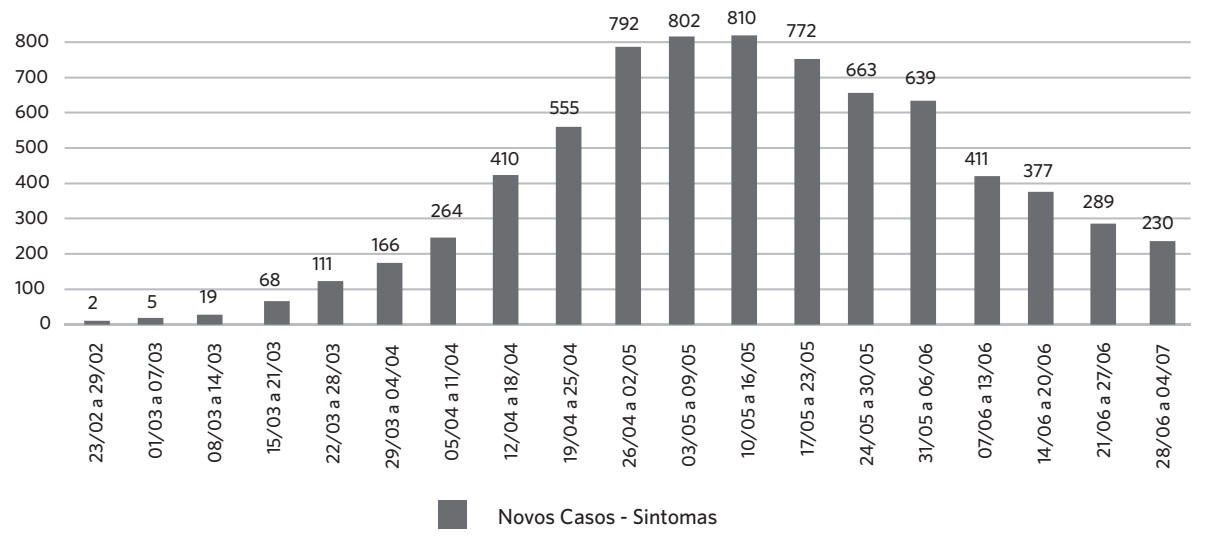

Fonte: Elaboração própria. 
Gráfico 3. Distribuição de óbitos por Covid-19 por semana epidemiológica, Niterói, 2020

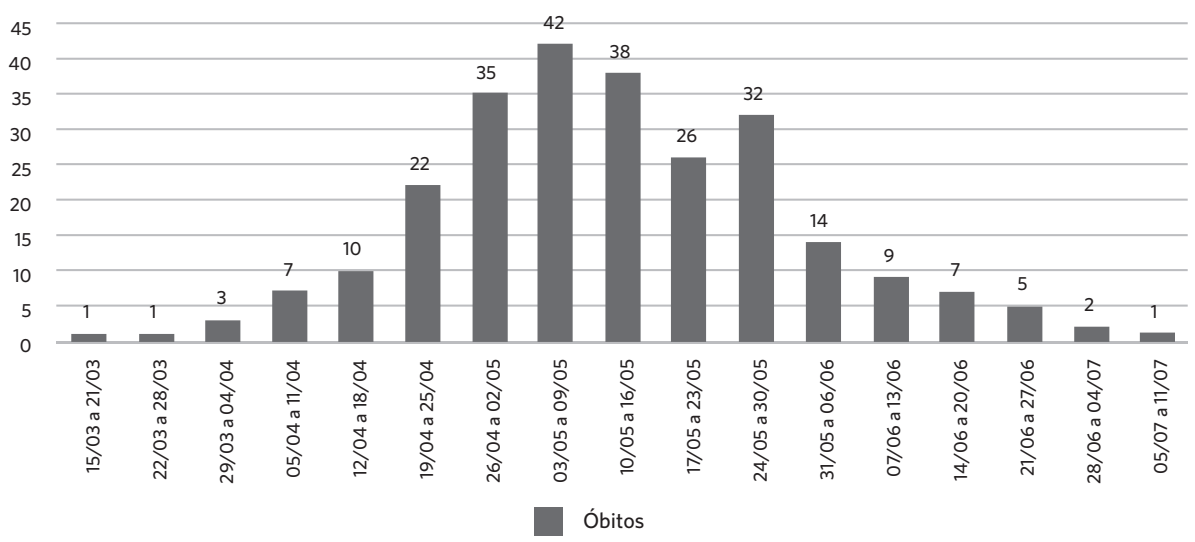

Fonte: Elaboração própria.

Os dados da evolução da Covid-19 por Semana Epidemiológica (SE) evidenciaram um importante panorama quanto à propagação da doença e à letalidade no município. Os novos casos, de acordo com a data dos primeiros sintomas (gráfico 2), permitem observar que a doença na cidade de Niterói teve seu pico entre as SE 18 e 21 (isto é, entre os dias 26 de abril e 23 de maio), em que alcançou uma média semanal próxima a 800 novos casos por semana. Desde a semana 21, transcorreram praticamente dois meses com quedas consistentes no número de novos casos, atingindo um patamar de cerca de um terço do total de casos do momento de pico da epidemia.
É importante ressaltar que tal queda ocorreu em um momento em que houve aumento na realização de testes na cidade, conforme observa-se no gráfico 4.

A análise dos óbitos (gráfico3) condizem com a afirmação feita acima, tendo seu pico ocorrido na SE 19 (ou seja, entre os dias 3 e 9 de maio), seguido de um repique na SE 22 ( 24 a 30 de maio de 2020), porém, mantendo o movimento decrescente desde então. $\mathrm{O}$ diagnóstico é complementado com a observação da redução no total de pacientes hospitalizados com Covid-19 em leitos clínicos e de UTI em hospitais públicos e privados, conforme observado no gráfico 5 .

Gráfico 4. Evolução do número de novos casos de acordo com a data dos sintomas e das notificações, Niterói, 2020

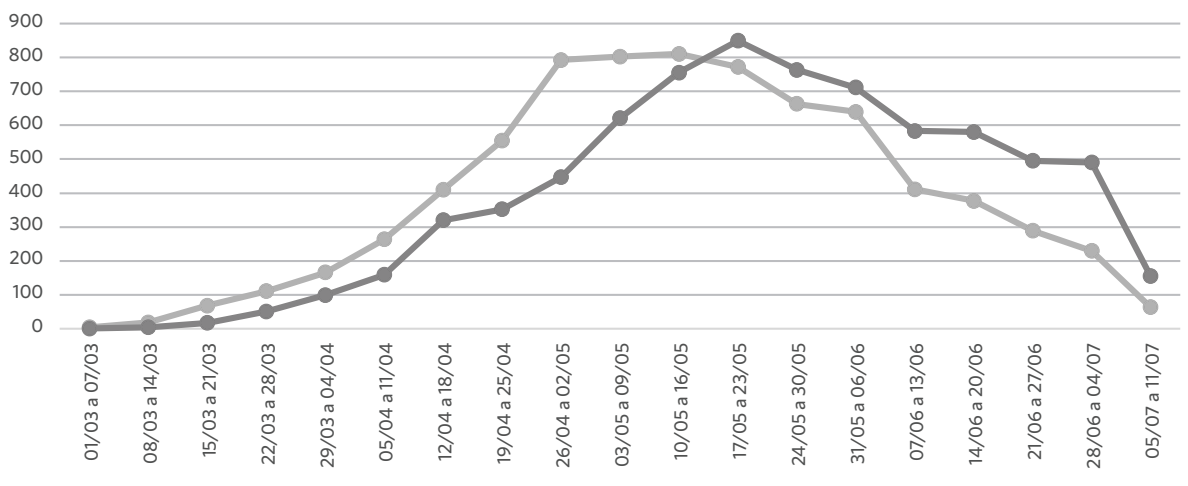


Gráfico 5. Evolução do número de pacientes internados com Covid-19 em leitos clínicos e de UTI da rede Privada e do SUS, Niterói, de 17/05 a 12/07/2020

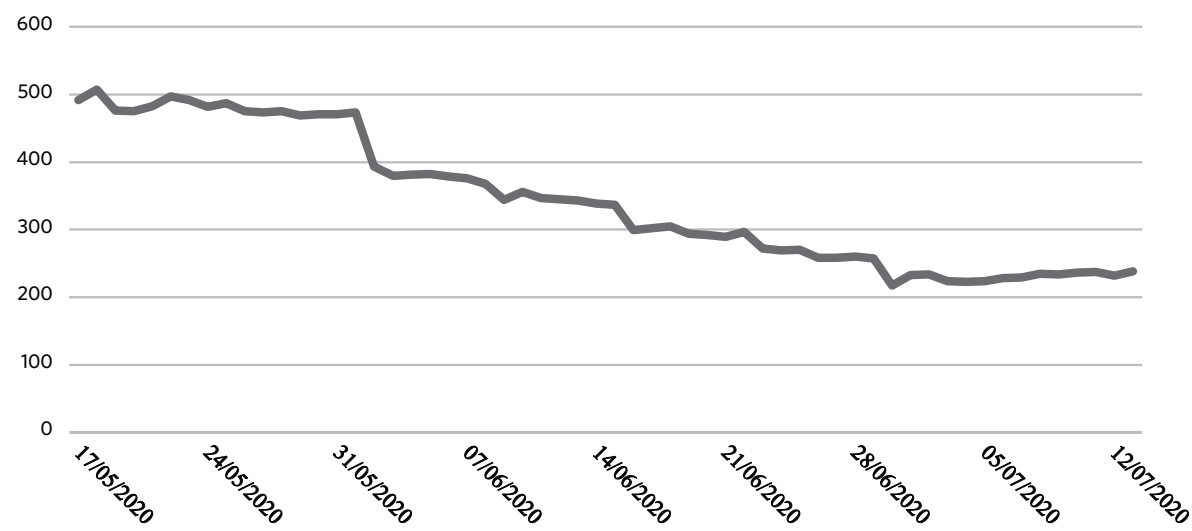

Fonte: Elaboração própria.

Esse cenário possibilitou o planejamento da retomada das atividades, coordenadas pelo gabinete de crise, desdobrando na construção do Plano de Transição para o Novo Normal ${ }^{32}$; garantindo a segurança e a manutenção da situação decrescente dos índices de propagação tem orientado a retomada de atividades a partir de (re)adequações e essencialidade aos diferentes setores da sociedade. As medidas de prevenção foram adotadas em um esforço coletivo de diminuição dos riscos de transmissibilidade, garantindo um processo de transição gradual das atividades, tendo como balizadora a avaliação de risco que pondera: a intensidade de contato, o número de contatos e o potencial de modificação, e de adaptação da atividade para reduzir os riscos de transmissibilidades. As ações orientadas nessa fase foram fundamentais para a contenção da pandemia, até que consigamos atingir a imunização massiva de toda a população.

\section{Considerações finais}

Pode-se inferir que as ações intersetoriais adotadas em Niterói se aproximaram do modelo de intersetorialidade ascendente proposto por Sposati ${ }^{16}$, uma vez que diferentes representações da gestão municipal construíram um conjunto de propostas com um objetivo em comum: evitar mortes e reduzir o impacto social causado pela Covid-19. A partir dos resultados exitosos das medidas de mitigação e da segunda fase de enfrentamento da pandemia, tornou-se possível o desenvolvimento de diferentes ações entre diversos setores, combinando distintos modelos de intersetorialidade"1 , na implementação do Plano de Transição Gradual para o Novo Normal, considerando, para tal, a atuação de sujeitos/técnicos dos diversificados setores sociais para a elaboração de propostas de forma colaborativa para o retorno das atividades em modo seguro.

Por fim, destaca-se a importância da comunicação e da garantia de acesso às informações que proporcionaram maior participação e adesão da sociedade ao plano de enfrentamento da Covid-19, sendo um fator central do êxito das medidas de mitigação. Estratégias estas que continua a produzir efeitos na coconstrução e planejamento da retomada das atividades, que vêm mantendo índices médios de $45 \%$ de isolamento social, além da adesão importante aos protocolos e medidas de segurança e prevenção nas situações de reabertura de setores. 


\section{Colaboradores}

Conceição MR (0000-0001-9696-9112)*

e Freire RP (0000-0003-4366-7123)* são responsáveis pela elaboração, concepção e desenho do estudo; análise e interpretação dos dados do trabalho; elaboração de versões preliminares do manuscrito e revisão crítica de importante conteúdo intelectual; e aprovação final da versão a ser publicada. Macário FC (0000-0002-4067-3942)*, Oliveira RAT (0000-0003-4939-017X)* e Franco CM (0000-0002-7401-1808)* são responsáveis pela revisão crítica de conteúdo intelectual e aprovação final da versão ser publicada.

\section{Referências}

1. Organização Mundial da Saúde. Novel Coronavirus (2019-nCoV) Situation Report - 1. 21 January 2020. [internet]. 2020 [acesso em 2020 jun 8]. Disponível em: https://www.who.int/emergencies/diseases/novel-coronavirus-2019/situation-reports.

2. Fundação Oswaldo Cruz. Organização Mundial da Saúde declara novo coronavírus uma pandemia [internet]. Rio de Janeiro: Fiocruz; 2020. [acesso em 2020 jun 10]. Disponível em: https://www.canalsaude.fiocruz.br/noticias/noticiaAberta/organizacao-mundial-da-saude-declara-novo-coronavirus-uma-pandemial1032020.

3. Brasil. Ministério da Saúde. Plano de Contingência Nacional para Infecção Humana pelo novo Coronavírus COVID-19. Brasília, DF: MS; 2020.

4. Smith JA. Vulnerability and the power of privilege in a pandemic. Health Promot J Austral. 2020; (31):158160

5. Walker PGT, Whittaker C, Watson O, et al. The Global Impact of COVID-19 and Strategies for Mitigation and Suppression. Imperial College London [internet]. 2020 [acesso em 2020 jun 7]. Disponível em: https://doi.org/10.25561/77735.
6. Akerman M, Franco de Sá R, Moyses S, et al. Intersetorialidade? IntersetorialidadeS! Ciênc. Saúde Colet. 2014; 19(11):4291-4300.

7. Ferreira VSC, Silva LMV. Intersetorialidade em saúde: um estudo de caso. In: Hartz ZMA, Silva LMV, organizadores. Avaliação em saúde: dos modelos teóricos à prática na avaliação de programas e sistemas de saúde. Salvador: EDUFBA; Rio de Janeiro: Fiocruz, 2005. p.103-150.

8. Inojosa RM. Sinergia em políticas e serviços públicos: desenvolvimento social com intersetorialidade. Cad. Fundap. 2001; (22):102-110.

9. Mendes R, Akerman M. Intersetorialidade: reflexões e práticas. In: Fernandez JCA, Mendes RB, organizadores. Promoção da Saúde e Gestão Local. São Paulo: Hucitec; Cepedoc; 2007. p. 85-110.

10. Junqueira LAP, Inojosa RM. Desenvolvimento social e intersetorialidade: a cidade solidária. São Paulo: Fundap; 1997.

11. Nascimento S. Reflexões sobre a intersetorialidade entre as políticas públicas. Serviço Soc. Socied. 2010; (101):95-120. 
12. Instituto Brasileiro de Geografia e Estatística. Dados de Niterói. [internet]. 2020 [acesso em 2020 jul]. Disponível em: https://cidades.ibge.gov.br/brasil/rj/niteroi/panorama.

13. Brasil. Ministério da Saúde, Departamento de Informática do Sistema Único de Saúde. DATASUS [internet]. Brasília, DF: MS; 2020. [acesso em 2020 jul 9]. Disponível em: http://www2.datasus.gov.br/DATASUS/index.php?area=02.

14. Niterói. Prefeitura. Decreto Municipal no 13.506 de 16 de março de 2020. Dispõe sobre a declaração de emergência em saúde pública no Município de Niterói devido a pandemia de doença infecciosa viral respiratória - COVID-19, causada pelo agente Novo Coronavírus - SARS-CoV-2 [internet]. 2020 [acesso em 2020 jul 8]. Disponível em: https://leismunicipais. com.br/a/rj/n/niteroi/decreto/2020/1350/13506/ decreto-n-13506-2020-dispoe-sobre-a-declaracao-de-emergencia-de-saude-publica-decorrente-da-pandemia-do-coronavirus-sobre-a-suspensao-de-aulas-na-rede-publica-municipal-de-niteroi-de-16-a-31-de-marco-sobre-as-medidas-de-enfrentamento-e-da-outras-providencias.

15. Niterói. Prefeitura. Decreto Municipal no 13.505 de 16 de Março de 2020. Cria Gabinete de Crise para centralizar a tomada de decisões, integrar e alinhar as iniciativas do Município na prevenção e assistência à população em relação à disseminação do vírus COVID-19 no Município de Niterói e dá outras providências. [internet] 2020 [acesso em 2020 jul 8]. Disponível em: https://leismunicipais. com.br/a/rj/n/niteroi/decreto/2020/1350/13505/ decreto-n-13505-2020-cria-gabinete-de-crise-para-centralizar-a-tomada-de-decisoes-integrar-e-alinhar-as-iniciativas-do-municipio-na-prevencao-e-assistencia-a-populacao-em-relacao-a-disseminacao-do-virus-covid-19-no-municipio-de-niteroi-e-da-outras-providencias.

16. Sposati A. Gestão pública intersetorial: sim ou não? Comentários de experiência. Serv Soc. Socied. 2006; (85):133-141.

17. Word Health Organization. Infection Prevention and Control guidance for Long-Term Care Facilities in the context of COVID-19. Interim guidance. [internet]. 2020. [acesso em 2020 jul 8]. Disponível em: https://www.who.int/publications/i/item/infection-prevention-and-control-for-long-term-care-facilities-in-the-context-of-covid-19.

18. Portugal. Secretaria Regional de Saúde. Despacho ${ }^{\circ}$ 385/2020 de 13 de março de 2020 - Declaração de Situação de Alerta - COVID-19. [internet]. 2020. [acesso em 2020 jul 7]. Disponível em: https://jo.azores.gov. pt/\#/ato/b238154b-7859-42a2-ab50-4d4a00ea4858.

19. Itália. Consigli dei Ministri. Misure urgenti di di contenimento del contagio sull'interno territorio nazionale [internet]. 2020 [acesso em 2020 jul 15]. Disponível em: https://www.esteri.it/mae/resource/ doc/2020/03/decreto_9_marzo.pdf.

20. Espanha. Ministerio de la Presidencia. Relaciones con las Cortes y Memoria Democrática [internet]. 2020 [acesso em 2020 jul 20]. Disponível em: https://www.boe.es/buscar/pdf/2020/BOE-A-2020-3692-consolidado.pdf.

21. Souza DO, Silva EVS, Silva NO. Determinantes Sociais da Saúde: reflexões a partir das raízes da "questão social”. Saúde Soc. 2013; 22(1):44-56.

22. Buss PM, Pellegrini AF. A saúde e seus determinantes. PHYSIS: Rev. Saúde Colet. 2007; 17(1):77-93.

23. Niterói. Prefeitura. Decreto Municipal no 13.564 de 2 de Abril de 2020. Regulamenta o Fundo de Crédito Emergencial do Município de Niterói - Niterói Supera, instituído pela Lei ${ }^{\circ} 3.481$ de 2 de abril de 2020, criado no contexto das medidas para o enfrentamento econômico da epidemia da COVID-19 [internet]. 2020 [acesso em 2020 jul 30]. Disponível em: https://leismunicipais.com.br/a/rj/n/niteroi/ decreto/2020/1356/13564/decreto-n-13564-2020-regulamenta-o-fundo-de-credito-emergencial-do-municipio-de-niteroi-niteroi-supera-instituido-pela-lei-n-3481-de-2-de-abril-de-2020-criado-no-contexto-das-medidas-para-o-enfrentamento-economico-da-epidemia-da-covid-19. 
24. Niterói. Prefeitura. Decreto Municipal no 13.538 de 3 de Abril de 2020. Regulamenta o Programa Empresa Cidadã de Niterói, instituído pela Lei ${ }^{\circ}$ $3.482 / 2020$, criado no contexto do enfrentamento aos efeitos econômicos do COVID19. Rio de Janeiro: Niterói. [internet]. 2020 [acesso em 2020 jul 27]. Disponível em: https://leismunicipais. com.br/al/rj/n/niteroi/decreto/2020/1353/13538/ decreto-n-13538-2020-regulamenta-o-programa-empresa-cidada-de-niteroi-instituido-pela-lei-n-3482-2020-criado-no-contexto-do-enfrentamento-aos-efeitos-economicos-do-covid19.

25. Niterói. Prefeitura. Decreto Municipal no 13.526 de 26 de Março de 2020. Regulamenta a concessão do benefício instituído pela Lei $\mathrm{n}^{\circ} 3477$, de 24 de março de 2020 aos Microempreendedores Individuais - MEI inscritos no cadastro da Secretaria Municipal de Fazenda de Niterói até $1^{\circ}$ de março de 2020. Rio de Janeiro: Niterói. [internet]. 2020 [acesso em 2020 jul 13]. Disponível em: https://leismunicipais. com.br/a/rj/n/niteroi/decreto/2020/1352/13526/ decreto-n-13526-2020-regulamenta-a-concessao-do-beneficio-instituido-pela-lei-n-3477-de-24-de-marco-de-2020-aos-microempreendedores-individuais-mei-inscritos-no-cadastro-da-secretaria-municipal-de-fazenda-de-niteroi-ate-1-de-marco-de-2020.

26. Niterói. Prefeitura. Decreto Municipal $n^{\circ} 13.570$ de 24 de Abril de 2020. Cria grupo de trabalho para apoio à retomada das atividades econômicas [internet]. 2020 Abr 26 [acesso em 2020 jul 15]. Disponível em: https://leismunicipais.com.br/a/rj/n/niteroi/decreto/2020/1357/13570/decreto-n-13570-2020-cria-grupo-de-trabalho-para-apoio-a-retomada-das-atividades-economicas.

27. Brasil. Ministério da Saúde. Portaria $n^{\circ} 1.559$, de $1^{\circ}$ de agosto de 2008. Institui a Política Nacional de Regulação do Sistema Único de Saúde - SUS. Diário Oficial da União. 2 Ago 2008.

28. Brasil. Ministério da Saúde. Medidas não-farmacológicas [internet]. 2020 [acesso em 2020 jul 25]. Disponível em: https://coronavirus.saude.gov.br/index. php/medidas-nao-farmacologicas.
29. Word Health Organization. WHO Director-General's opening remarks at the media briefing on COVID-19 - 16 March 2020 [internet]. 2020 [acesso em 2020 jul 30]. Disponível em: https://www.who.int/dg/speeches/detail/who-director-general-s-opening-remarks-at-the-media-briefing-on-covid-19---16-march-2020.

30. Brasil. Ministério da Saúde. Protocolo de manejo clínico do coronavírus (covid-19) na atenção primária à saúde [internet]. 2020 [acesso em 2020 jul 18]. Disponível em: https://portalarquivos.saude.gov.br/images/pdf/2020/April/08/20200408-ProtocoloManejo-ver07.pdf.

31 Niterói. Prefeitura. Linha do tempo - Ações da Prefeitura para combate ao coronavírus 2020 [internet]. 2020 [acesso em 2020 jul 27]. Disponível em: http:// www.niteroi.rj.gov.br/index.php?option=com_conte nt\&view=article\&id=6505:2020-03-18-20-02-10.

32. Niterói. Prefeitura. Decreto $n^{\circ} 13.604$ de 21 de Maio de 2020. Institui o Plano de Transição Gradual para o Novo Normal - Distanciamento Responsável para fins de prevenção e de enfrentamento à epidemia causada pelo novo Coronavírus (COVID-19) no âmbito do Município de Niterói [internet]. 2020 [acesso em 2020 jul 30]. Disponível em: https://leismunicipais. com.br/a/rj/n/niteroi/decreto/2020/1361/13604/ decreto-n-13604-2020-institui-o-plano-de-transicao-gradual-para-o-novo-normal-distanciamento-responsavel-para-fins-de-prevencao-e-de-enfrentamento-a-epidemia-causada-pelo-novo-coronavirus-covid-19-no-ambito-do-municipio-de-niteroi.

33. Niterói. Prefeitura, Secretaria Municipal de Saúde. Sistema de Gestão da Geoinformação da Prefeitura Municipal de Niterói [internet]. SIGeo-Niterói. 2020 [acesso em 2020 jul 30]. Disponível em: https://experience.arcgis.com/experience/305269f3cdd 2483 9b263c5ab346elaa7.

\footnotetext{
Recebido em 21/07/2020

Aprovado em 01/09/2020

Conflito de interesses: inexistente
}

Suporte financeiro: não houve 